\title{
Memory Construction, Suggestibility Effect And Eyewitness: From Laboratory To Legal Contexts
}

Pino O

Department of Neurosciences, Piazzale San Francesco, 2 - Parma, Italy.

Keywords: Memory; Suggestibility; Eyewitness memory; Forgetting; Interrogation.

\section{*Corresponding Author:}

Olimpia Pino,

Department of Neurosciences, Piazzale San Francesco, 2 - Parma, Italy. E-mail: olimpia.pino@unipr.it

Recieved: March 05, 2015

Published: March 10, 2015

Citation: Pino O (2015) Memory Construction, Suggestibility Effect And Eyewitness: From Laboratory To Legal Contexts. Int J Forensic Sci Pathol. 3(1e), 1-2. doi: http://dx.doi.org/10.19070/2332-287X-150003e

Copyright: Pino $\mathbf{O}^{\circ}$ 2015. This is an open-access article distributed under the terms of the Creative Commons Attribution License, which permits unrestricted use, distribution and reproduction in any medium, provided the original author and source are credited.

\section{Introduction}

One of the most intriguing debates in psychology concerns the permanence and accuracy of recovered memories. Yet, despite a prodigious level of research activity, our understanding of the interaction between old and new memories or between memories and new experience remains relatively incomplete. A topic of traditional interest for both theoretical and applied aspects of memory has been the study of eyewitness suggestibility. Witness' memory performance is affected by the fact that they retell their statement multiple times, for example to the police or other personnel in the justice system and to their family or friends. The notion of interrogative suggestibility usually designate "the extent to which, within a closed social interaction, people come to accept messages communicated to them during formal questioning, and as a result their behavioral response is affected in such a way as to either accept or resist suggestion" [1]. Not only how many times a recall is shared (repetition), but also how an event is shared influence the memory of that event.

The primary characteristic of memory mechanisms that contribute to suggestibility effect is its reconstructive nature: remembering appears to be far more definitively an affair of construction rather than one of simple reproduction [2]. Bartlett showed that when requested to recall The War of the Ghosts with repeated recall either from one individual to another or serial recall by the same subject, participants tended to incur in characteristic errors:
1. Omission (for peripheral details or elements outside the readers' cultural experience);

2. Rationalization;

3. Transformation of detail (from the atypical to the usual);

4. Changing the order of events.

Because of these observations, he proposed that retention follows a schematic organization. So, memories for events are reconstructed in the light of relevant available schemata. If leading questions allow rationalization or translation from the unusual to the conventional, it is not difficult for a witness incorporate these suggestions in her/his event remembering, thus creating it schema-consistent. It is plausible that any procedure that involves the asking of questions about an event could generate those circumstances that promote distortion effects. Such situations range from the employment interview, to the interrogation of suspects and witnesses to crimes, to how autobiographical material is solicited in clinical and forensic settings.

\section{Hypotheses, Experimental Paradigms and Fac- tors Involved In Eyewitness Memory}

Relevant for an accuracy-oriented approach, confidence and accuracy in eyewitness memory have been addressed in many studies [3-4] looking at factors that affect what individuals report, the level of specificity and the goal dependent criteria adopted in the response decision. Through the Post-Event Information paradigm (PEI), Elizabeth Loftus experimentally confirmed the effect of "misleading questions" on remembering of a witnessed event [5-6] indicating the situations in which incorrect information can become embedded or overwrite in the memory trace and, consequently, in the report of an event. This destructive updating of memory is responsible for what is defined misinformation effect.

McCloskey and Zaragoza [7] argued that there is no decisive evidence that misinformation completely dissolves memory for original material. Rather the occurrence of post-event information alters original memory; they suggested that both memories coexist and that the conditions that prevail at retrieval determine what form will recovered. In this perspective, misinformation effect is described in terms of retrieval accessibility without recourse to blending or superimposition mechanisms (coexistence hypoth- 
esis), or of misattribution of source memories [8].

Under some circumstances factors such as expectations, demand characteristics or the negative nature of the event can influence the report of misinformation [9]. A potential mechanism underlying misinformation effect can be the retrieval-induced forgetting [10] due to the active suppression or inhibition of undesired material at retrieval. Usually the retrieval cues are insufficiently specified and therefore they access the desired material but also related unwanted material. The fact that retrieval-induced forgetting is probable to occur where questions have been asked about a subset of items known about an event is of theoretical interest and potentially of applied importance in understanding when misinformation effects are most likely to happen. Most of police interviews, for example, do not encompass exhaustive retrievals for information about a witnessed event. Occasionally this may be because the investigating police officer is interested in current investigative goals that define those aspects of the event are considered most important. In other cases, there may be insufficient time to carry out exhaustive interviews, or the police officer may fail to understanding the complexity of what has occurred. Those details that were not the subject of initial retrieval could in time become critical aspects and could be more poorly recalled during any subsequent retrieval session. The incomplete retrieval of information about an event could produce those conditions that encourage the likelihood of misinformation about items that initially have not been retrieved.

Eye witness' confidence appears the most influential factor in the forensic process for the evaluation of the correctness of eyewitness memory reports. Confidence accuracy refers to the match between a person's confidence judgments and the correctness of her/his answers (or Meta cognitive realism). It was indicated that different portions of witness' memory statements, such as information about actions (and not the information about details), may have a high degree of recall properties and meta-cognitive accuracy [11]. Free recall and focused questions are different in cognitive demand because focused questions involve recognition and free recall test participants' own-regulated recall. Therefore, individual' confidence accuracy can vary for action and detail information depending on question type. This aspect of eyewitnesses' early free recall can have an important impact on the following course of the forensic investigation.

\section{Conclusion}

There is a growing body of literature proposing exposure to life adversity in later psychological vulnerability in adulthood; particularly, low self-esteem, susceptibility to negative emotional states [12] and interrogative suggestibility [13]. This studies indicate that interviewees reporting a high number of association between the reported experience of negative life events (NLEs) could be more vulnerable (because they may experience a heightened state of uncertainty) to police interrogative pressure/critical feedback and become ever more likely to answer shifting in their responses. This suggests that, in the occurrence of real-life stressors, interrogative vulnerability may be a problem for interviewees with a high number of NLEs. This issue needs further research.

More recently, in order to distinguish suggestibility that occurs incidentally from suggestibility that occurs due to interrogative pressure it was proposed the use of the expression of investigative suggestibility [14]. The benefits of free recall and the threats for misleading questions are reflected in procedures advocated in modern questioning techniques for vulnerable witnesses, such as the NICHD protocol [15].

\section{References}

[1]. Gudjonsson GH (1992) Interrogative suggestibility: Factor analysis of the Gudjonsson Suggestiblity Scale (GSS 2). Personality and Individual Differences, 13: 479-481.

[2]. Bartlett FC (1932) Remembering: A Study in Experimental and Social Psychology. Cambridge: Cambridge University Press.

[3]. Koriat A, Goldsmith M (1996) Monitoring and control processes in the strategic regulation of memory accuracy. Psychological Review, 3:490-517.

[4]. Koriat, A, Goldsmith, M, \& Pansky, A (2000) Towards a psychology of memory accuracy. Annual Review of Psychology, 51: 481-537.

[5]. Loftus EF (1979) Eyewitness testimony. Cambridge, MA: Harvard University Press.

[6]. Loftus EF, Miller DG, Burns HJ (1978) Semantic integration of verbal information into a visual memory. Journal of Experimental Psychology: Human Learning \& Memory, 4:19-31.

[7]. McCloskey M, Zaragoza M (1985) Misleading post event information and memory for events: Arguments and evidence against memory impairment hypotheses. Journal of Experimental Psychology: General,114:1-16.

[8]. Lindsay DS, Johnson M (1989) The eyewitness suggestibility effect and memory for source. Memory and Cognition, 17:349-358.

[9]. Paz-Alonso PM, Goodman GS, Ibabe I (2013) Adult eyewitness memory and compliance: Effects of post-event misinformation on memory for a negative event. Behavioral Sciences and the Law, 31: 541-558.

[10]. Anderson MC, Bjork RA, Bjork EL (1994) Remembering can cause forgetting: Retrieval dynamics in long-term memory. Journal of Experimental Psychology: Learning, Memory, and Cognition, 20:1063-1087.

[11]. Sarwar F, Allwood CM, Ker AI (2014) Effects of different types of forensic information on eyewitness' memory and confidence accuracy. The European Journal of Psychology Applied to Legal Context 6: 17-27.

[12]. Rosenman S, Rodgers B (2006) Childhood adversity and adult personality. Australian and New Zealand Journal of Psychiatry, 40: 482-490.

[13]. Drake K, Bull R (2011) Individual differences in interrogative suggestibility: Life adversity and field dependence, Psychology, Crime \& Law, 17: 8, 677-687.

[14]. Ridley AM, Gabbert F, La Rooy DJ (2013) Suggestibility in legal contexts: Psychological research and forensic implications, John Wiley \& Sons, Ltd.

[15]. Lamb ME, Hershkowitz I, Orbach Y, Esplin PW (2008) Tell me what happened: Structured investigative interviews of child victims and witnesses. Chichester: John Wiley \& Sons, Ltd. 